\title{
Spontaneous Extrusion of a Reversed Internal Magnet of a Cochlear Implant
}

\author{
Ji-A Kim, MD, Sung-Won Choi, MD, PhD, Se-Joon Oh, MD, PhD and Soo-Keun Kong, MD, PhD \\ Department of Otorhinolaryngology and Biomedical Research Institute, Pusan National University Hospital, Busan, \\ Korea
}

\begin{abstract}
- ABSTRACT -
With the increasing of cochlear implantation, necessity of magnetic resonance imaging (MRI) of cochlear implant $(\mathrm{CI})$ recipients is also increasing. This patient was previously reported as a case of reversion of internal magnet of CI after undergoing MRI. At that time, the CI device functioned normally after the polarity reversion of external device. Eight years later from that event, the patient came for sudden functional loss of the CI. Then, we found the patient's internal magnet of CI was spontaneously extruded through the skin. The function of CI was perfectly recovered after the surgery for insertion of internal magnet. (J Clinical Otolaryngol 2021;32:228-232)
\end{abstract}

KEY WORDS: Cochlear implants; Magnets; Displacement; Magnetic resonance imaging; Complication.

\section{Introduction}

Currently, cochlear implants (CIs) are considered an effective method to manage severe-to-profound hearing disturbances, and their use has been increasing worldwide. ${ }^{1)}$ Magnetic resonance imaging (MRI) is required in many cases, such as acute head trauma or malignancy, even in CI recipients.

MRI uses various strengths $(1.5-3 \mathrm{~T})$ of magnetic field to produce images, and CI uses a magnet to connect internal and external devices. It is assumed that damage may be caused by interference with the implant in electromagnetic fields. Interference could destroy the implant, dislocate the inner magnet, and the resulting heat could damage the surrounding tissue. ${ }^{2,3)}$

In the early days, MRI was contraindicated in CI recipients because of risk of complication. ${ }^{4)}$ Recently, with the development of MRI-compatible CI, MRI has become more feasible. However, their feasibility for
MR depends on the CI device and MRI scan site. ${ }^{2,5,6)}$

Nevertheless, many complications have been reported in CI recipients after undergoing MRI. These complications include discomfort or pain during the MR scan, magnet displacement, total demagnetization (implant malfunction), and reversing the polarity. ${ }^{6-10)}$ To prevent these problems, gauze bandage or head protection at appropriate site is recommended. ${ }^{3,9)}$

In 2014, a case of a reversed internal magnet of CI after MRI was reported. ${ }^{11)}$ Here, we have reported the case of the same patient in which the reversed magnet of CI spontaneously extruded from the skin.

\section{Case Report}

An 81-year-old woman who had undergone cochlear implantation in the left ear with Nucleus 24R Contour Advance (Cochlear, Sydney, Australia) in 2005 presented with sudden CI dysfunction. She reported sudden

Received: August 26, 2021 / Revised: September 23, 2021 / Accepted: October 27, 2021

Corresponding author: Soo-Keun Kong, Department of Otorhinolaryngology and Biomedical Research Institute, Pusan National University Hospital, 179 Gudeok-Ro, Seo-gu, Busan 49241, Korea

Tel: +82-51-240-7332 · Fax: +82-51-246-8668·E-mail: entkong@gmail.com 
detachment of the external device of the CI and inability to hear sounds despite her manually attaching the external device. At presentation in our Ear, Nose, and Throat Clinic, the internal magnet had come out and was attached to the external device of the CI, leaving only a skin scar without open wounds or fistulas (Fig. 1). She had no discomfort at the scar area.

She had an unusual history of reversion of the internal magnet of the CI after undergoing MRI in 2012. ${ }^{11)}$ It was 1.5T lumbar spine MRI to evaluate her back pain, and she didn't head bandage before the MR scan. At that time, after we confirmed the reversion of only the internal magnet with no displacement of the internal device, we reversed the polarity of the external magnet. Before and after the event in 2012, her auditory performance had been excellent, with a category of auditory performance of 7 . In the subsequent 7 years of follow-up at intervals of 1 to 3 months, she had no complaints. However, 8 years after the previous event, the inverted internal magnet spontaneously extruded through the skin.

Exploration surgery was performed to check the condition of the internal device. Intraoperatively, we found that the silicone cover for fixation of the internal magnet of the inner device was intact, without displacement of the inner device (Fig. 2A). During the surgery, we checked that the magnet could move freely within the silicone bed, and we found it almost impossible to stand upright or turn over with the magnet fully inserted in the silicone bed. Then, we reinserted the internal magnet, placed a silicone cover over it, and fixed it (Fig. 2B). As the skin covering the internal magnet had strong scar tissue with no other fistulas, so we didn't perform additional skin reinforcement. At 3 weeks postoperatively, the CI worked normally, and her auditory performance had been fully restored (Fig. 3).

This study was approved by the institutional review board of Pusan National University Hospital (IRB No. 2104-012-101).

\section{Discussion}

When the CI recipients complain of a sudden loss of CI function, we should first check if there is a problem with the device. In this case, there was a problem with the CI device, but this wasn't a simple mechanical failure. Fortunately, the patient came to our ENT clinic with the extruded internal magnet attached to the external device (Fig. 1). However, it was also possible that the internal magnet was loss after the extrusion. A key
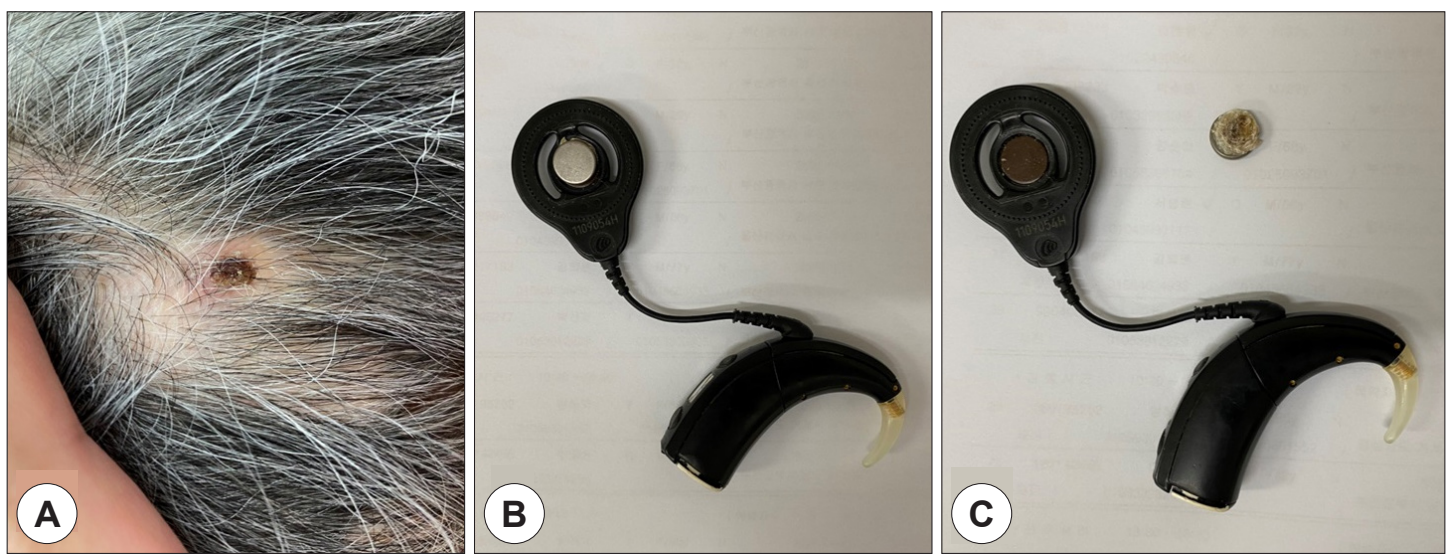

Fig. 1. Spontaneously extruded internal magnet confirmed in the outpatient ear, nose, and throat clinic. A : Remnant scar on the patient's scalp. B : Extruded internal magnet attached to the external device. C : Extruded internal magnet detached from the external device. 

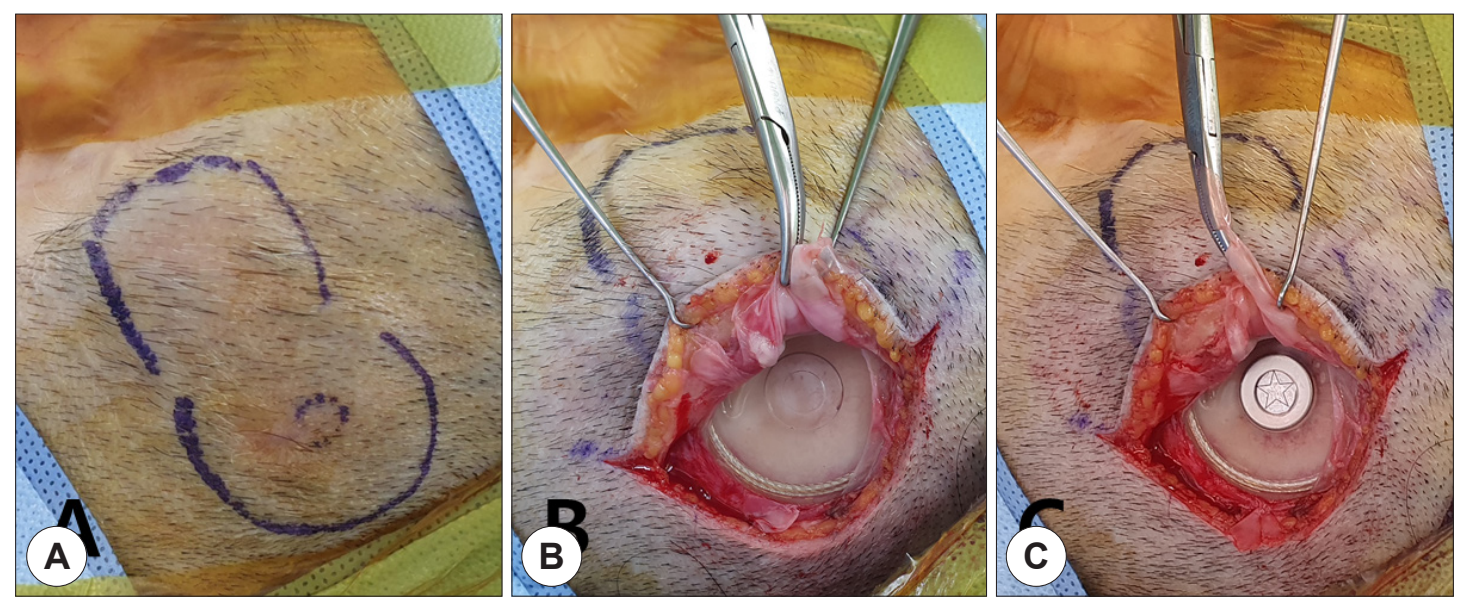

Fig. 2. Intraoperative findings. A : The skin overlying the internal device without fistulas or scars. B : Internal device without an internal magnet. C : Internal device with the correctly reinserted internal magnet.

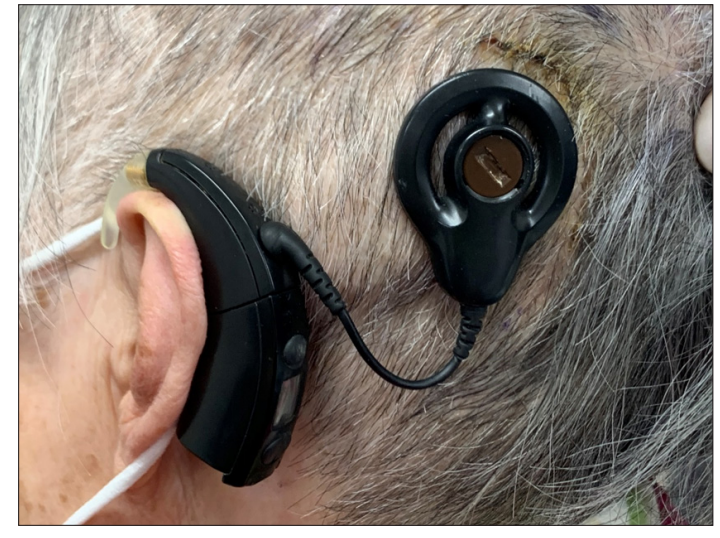

Fig. 3. One-month postoperative follow-up.

factor is the patient's previous history of magnet reversion after undergoing the MRI.

As we mentioned in a previous report of this patient, she experienced severe pain in the CI implanted site in the left during MRI. She felt the button-sized material vertically against her skull, and compressed it with her hand to reduce the pain. ${ }^{11)}$ Subsequently, the pain was remarkably reduced, but her CI ceased to work. On $\mathrm{X}$-ray, abnormal displacement findings were not shown. We concluded that the internal magnet was turned over, and the CI function was restored by reversing the polarity of the external magnet.
A few articles have been published on the case of internal magnet reversion in CI after MRI. ${ }^{3,8,12)}$ They used X-ray to evaluate the magnet dislocation. Out of them, Jeon and Crane revered the polarity of external device to restore the CI function and there is no report about the long-term complication of this polarity reversal. And only Ozturk conducted surgical management to check the position of internal magnet and magnet housing. In a rare case of vertical displacement of internal magnet after MRI, Di Nardo restored the magnet in correct place in correct orientation by bimanual noninvasive method. ${ }^{13)}$ When restoring, Di Nardo checked not only the orientation of magnet, but also magnet housing in silicone pocket.

Herein, we reported a rare long-term complication of polarity reversal. To prevent another case of same complication, it is very important to find out the cause and mechanism of this complication.

We came up several hypotheses explaining the spontaneous extrusion of internal magnet. First, excessive magnet strength made a skin thinned over time. Second, when the magnet was turned over in 2012, overlying skin was damaged and inflammation induced. And following chronic inflammation caused the thinning of the 
skin. Third, the internal magnet wasn't in stable pocket, inside the silicone cover, and the instability of internal magnet induces skin thinning over a long period of time.

As reported in 2012, ${ }^{11)}$ the CI function was restored by reversing the polarity of the external magnet. And, reversing the polarity means not a change of power, but a change of vector. Therefore, it is hard to think that there was significant increase in magnetic force after the polarity reversal, and the first hypothesis is unlikely.

After that event, the patient came to our ENT clinic at intervals of 1 to 3 months for 7 years. She complained of discomfort and slight redness in that area at first visit, but since then she has never complained of any inflammatory symptoms. So, there wasn't chronic inflammation at the magnet reversion site, and the second hypothesis is also unlikely.

From what we checked during the surgery, it is almost impossible to move the internal magnet within the silicone bed. The silicone bed was designed to reliably hold the internal magnets in place, although the magnet can be removed when needed. The silicone cover has a hole smaller than the magnet, and the silicone cover is elastic, so the hole gets bigger when we move the magnet inside to stand up or take it out. When the patient of this report felt pain during the MR scan in 2012, she just pressed the painful area by her one hand. This is a crucial difference between this case and Di Nardo's. ${ }^{13)}$

During the MR scan, the internal magnet is subjected to forces by a magnetic field. Eerkens reported main cause for magnet dislocation is rotational force in magnetic field of MRI. ${ }^{14)}$ These rotational forces could cause the internal magnet to move in various directions, moving the center or axis of the magnet to a different position. Like figures in Jeon's report, ${ }^{12)}$ magnet reversal might occur through vertical displacement with partial extrusion from silicone bed. Actually there is one case of vertical displacement of internal magnet after MRI. ${ }^{13)}$ Therefore, given these conditions, it could be possible that the reversed magnet wasn't fully anchored in the silicone bed at the first reversal event. Especially in this case, magnet is positioned in vertical direction at first, and reversed while the patient pressed with her one hand without any confirmation of axis or center of the magnet. In other words, the magnet was located in internal device pocket, but not inside the silicone bed, just over the silicone cover, and it made the magnet unstable under the skin. If the internal magnet is unstable, vibration or torsional movement of the magnet could be induced especially when the patient attaches or detaches the external devices. And, we thought that these mechanical irritations could thin the skin over a long period of time, about 8 years. Therefore, we believe the third hypothesis is more likely.

Compressive bandage before MRI is recommended to prevent these magnet-related complications in CI recipients. ${ }^{13,15)}$ However, even after compressive bandaging, complications related to internal magnet displacement or reversal have been reported. ${ }^{12,16,17)}$ In Crane groups' study, all CI recipients with mold material and gauze bandaging showed no CI malfunction or magnet displacement after MRI. One patient was unable to tolerate the compressive bandaging because of discomfort and internal magnet reversal occurred after MRI. ${ }^{3)}$

From these, we have been able to draw several conclusions. First, compression in the right place with the right pressure is critical. Or, mold for internal device with bandage like Crane's report ${ }^{3}$ could be recommended. Therefore, the patients and all concerned individuals should be trained to conduct compressive bandage correctly before MRI. Or, alternatively, the internal magnet should be removed before MRI. Second, if the internal magnet is reversed, surgical exploration should be conducted to check the orientation and housing in silicone bed of the internal magnet. Because simply reversing the polarity of the external magnet cannot guarantee that the internal magnet correctly positioned inside the silicone bed. Third, when a patient refuses exploration 
surgery, continued long-term follow-up for the overlying skin thickness is required.

\section{Acknowledgements}

This case report did not receive any specific grant from funding agencies in the public, commercial, or not-for profit sectors. No potential conflict of interest relevant to this article was reported.

\section{Funding Information}

Not applicable.

\section{Conflict of Interest}

No potential conflict of interest relevant to this article was reported.

\section{ORCID}

Ji-A Kim, https://orcid.org/0000-0001-9797-5135

Sung-Won Choi, https://orcid.org/0000-0002-7463-7720

Se-Joon Oh, https://orcid.org/0000-0001-8910-0064

Soo-Keun Kong, https://orcid.org/0000-0002-6783-3766

\section{Author Contribution}

Conceptualization: Kong SK.

Formal analysis: Kim JA.

Investigation: Kim JA.

Writing - original draft: Kim JA.

Writing - review \& editing: Kim JA, Choi SW, Oh SJ, Kong SK.

\section{Ethics Approval}

This study was approved by the institutional review board of $\mathrm{Pu}-$ san National University Hospital (IRB No. 2104-012-101).

\section{References}

1) Berrettini S, Baggiani A, Bruschini L, Cassandro E, Cuda D, Filipo R, et al. Systematic review of the literature on the clinical effectiveness of the cochlear implant procedure in adult patients. Acta Otorhinolaryngol Ital 2011;31(5):299-310.

2) Teissl C, Kremser C, Hochmair ES, Hochmair-Desoyer IJ. Magnetic resonance imaging and cochlear implants: compatibility and safety aspects. J Magn Reson Imaging 1999; 9(1):26-38.

3) Crane BT, Gottschalk B, Kraut M, Aygun N, Niparko JK. Magnetic resonance imaging at $1.5 \mathrm{~T}$ after cochlear implantation. Otol Neurotol 2010;31(8):1215-20.
4) Engler MB, Engler MM. The hazards of magnetic resonance imaging. Am J Nurs 1986;86(6):650.

5) Carlson ML, Neff BA, Link MJ, Lane JI, Watson RE, McGee KP, et al. Magnetic resonance imaging with cochlear implant magnet in place: safety and imaging quality. Otol Neurotol 2015;36(6):965-71.

6) Kim BG, Kim JW, Park JJ, Kim SH, Kim HN, Choi JY. Adverse events and discomfort during magnetic resonance imaging in cochlear implant recipients. JAMA Otolaryngol Head Neck Surg 2015;141(1):45-52.

7) Bawazeer N, Vuong H, Riehm S, Veillon F, Charpiot A. Magnetic resonance imaging after cochlear implants. J Otol 2019;14(1):22-25.

8) Öztürk E, Doruk C, Orhan KS, Çelik M, Polat B, Güldiken Y. A rare complication of cochlear implantation after magnetic resonance imaging: reversion of the magnet. J Craniofac Surg 2017;28(4):e372-4.

9) Özgür A, Dursun E, Beyazal Çeliker F, Terzi S. Magnet dislocation during 3T magnetic resonance imaging in a pediatric case with cochlear implant. Braz J Otorhinolaryngol 2019; 85(6):799-802.

10) Migirov L, Kronenberg J. Magnet displacement following cochlear implantation. Otol Neurotol 2005;26(4):646-8.

11) Kong SK, Oh SJ, Lee IW, Goh EK. The reversed internal magnet of cochlear implant after magnetic resonance imaging. Am J Otolaryngol 2014;35(2):239-41.

12) Jeon JH, Bae MR, Chang JW, Choi JY. Reversing the polarity of a cochlear implant magnet after magnetic resonance imaging. Auris Nasus Larynx 2012;39(4):415-7.

13) Di Nardo W, Giannantonio S, Schinaia L, De Corso E, Paludetti G. Noninvasive management of cochlear implant's inner magnet displacement after magnetic resonance. Laryngoscope 2013;123(3):783-6.

14) Eerkens HJ, Smits C, Hofman MBM. Cochlear implant magnet dislocation: simulations and measurements of force and torque at 1.5T magnetic resonance imaging. Ear Hear 2021; 42(5):1276-83.

15) Gubbels SP, McMenomey SO. Safety study of the Cochlear Nucleus ${ }^{\circledR} 24$ device with internal magnet in the 1.5 Tesla magnetic resonance imaging scanner. Laryngoscope 2006; 116(6):865-71.

16) Leong WJC, Yuen HW. Dislocation of cochlear implant magnet during 1.5 Tesla magnetic resonance imaging despite head bandaging, and its repositioning using an endoscopic approach. J Laryngol Otol 2018;132(10):943-5.

17) Cuda D, Murri A, Succo G. Focused tight dressing does not prevent cochlear implant magnet migration under 1.5 Tesla MRI. Acta Otorhinolaryngol Ital 2013;33(2):133-6. 